\title{
Clima organizacional e atividades de prevenção ao uso de risco de álcool'
}

\author{
Organizational climate and \\ alcohol risk prevention
}

\author{
Erica CRUVINEL ${ }^{2}$ \\ Telmo Mota RONZANI ${ }^{3}$
}

\section{Resumo}

O objetivo deste estudo foi avaliar a associação entre Clima Organizacional e atividades de prevenção ao consumo de álcool entre 97 profissionais da Atenção Primária à Saúde. O clima organizacional foi avaliado por meio de escala envolvendo os seguintes fatores: tomada de decisão, caos/stress e comunicação. As atividades de prevenção foram mensuradas a partir do número de Alcohol Use Disorders Identification Tests (instrumento de rastreamento) e de intervenções breves realizados no período de 6 meses após a capacitação presencial. Para verificar a existência das associações utilizou-se a correlação não paramétrica de Spearman, com 95\% de intervalo de confiança. O número de intervenções breves realizadas relacionou-se com Tomada de Decisão ( $p=0,337 ; p=0,001)$ e Comunicação $(p=0,281 ; p=0,005)$, enquanto o número de Alcohol Use Disorders Identification Tests aplicados associou-se com Tomada de Decisão $(p=0,288 ; p=0,004)$ e Comunicação $(p=0,215 ; p=0,035)$. Os resultados sugerem que ambientes com melhor percepção do clima organizacional podem ser facilitadores para a prevenção do uso de álcool na Atenção Primária à Saúde.

Unitermos: Abuso de álcool. Atenção primária à saúde. Clima organizacional. Prevenção.

\begin{abstract}
The aim of this paper was to evaluate the link between organizational climate and strategies to prevent alcohol consumption, among 97 Primary Health Care professionals. The organizational climate was measured by means of a scale involving the following factors: decision-making, chaos/stress and communication. Prevention activities were measured using AUDIT (screening test) and brief interventions carried out in the six-month period following the on-site qualification. The Spearman test was used, with a 95\% level of confidence, to assess correlation. The results showed a correlation between brief intervention and Decision Making ( $p=0.337 ; p=0.001)$ and Communication $(p=0.281 ; p=0.005)$. The number of AUDITs applied were also related to DM $(p=0.288 ; p=0.004)$ and Communication $(p=0.215 ; p=0.035)$. The results suggest that environments with better perceptions of Organizational Climate can enhance the prevention of alcohol use in Primary Health Care.
\end{abstract}

Uniterms: Alcohol abuse. Primary health care. Organizational climate. Prevention.

$\boldsymbol{\nabla} \mathbf{v} \boldsymbol{\nabla}$

1 Apoio: Conselho Nacional de Desenvolvimento Científico e Tecnológico, edital MCT- CNPq/ MS-SCTIE- DECIT - n 23/2006, processo 408753/2006-4.

2 Universidade Federal de Juiz de Fora, Polo de Pesquisa em Psicologia Social e Saúde Coletiva. R. José Lourenço Kelmer, s/n., Campus Universitário, 36036-900, Juiz de Fora, MG, Brasil. Correspondência para/Correspondence to: E. CRUVINEL. E-mail: <ecruvinel@yahoo.com.br>.

3 Universidade Federal de Juiz de Fora, Programa de Pós-Graduação em Saúde Coletiva e Psicologia. Juiz de Fora, MG, Brasil.

Agradecimentos: aos colegas do POPSS e profissionais de saúde que colaboraram com este estudo. 
O consumo abusivo de álcool está entre as principais causas evitáveis de morbimortalidade no mundo, sendo considerado fator de relação direta com problemas físicos, sociais e psicológicos, tais como câncer, distúrbios neuropsiquiátricos, cardiológicos e gastrintestinais, violência, acidentes e suicídio, entre outros (World Health Organization, 2007).

Dessa forma, diversos estudos vêm mostrando evidências substanciais dos benefícios da utilização de instrumentos de triagem (como o Alcohol Use Disorders Identification Test - Audit) associados a intervenções breves, em serviços de Atenção Primária à Saúde (APS), como forma de introduzir intervenções preventivas que venham a mudar a realidade epidemiológica do consumo de álcool (Bertholet, Daeppen, Wietlisbach, Fleming \& Burnand, 2005; Kaner et al., 2009; Ronzani, Mota \& Souza, 2009).

O Audit é um instrumento de rastreamento simples, composto de 10 perguntas, desenvolvido pela Organização Mundial da Saúde (OMS), como forma de identificar precocemente padrões de uso abusivo de álcool e problemas associados (Babor, Higgins-Biddle, Saunders \& Monteiro, 2001). Entretanto, apenas a identificação dos padrões de consumo não são suficientes para mudar a realidade epidemiológica do problema. Sendo assim, juntamente com esse instrumento, foram desenvolvidas estratégias terapêuticas simples intervenção breve, acessíveis a profissionais de diversas formações que atuam em serviços de APS (Babor \& Higgins-Biddle, 2001).

Estudos anteriores sugerem que a efetividade da intervenção breve e seu desempenho sejam idênticos ou até mesmo superiores aos de outras intervenções que demandam maior tempo e treinamento mais intensivo dos profissionais (Kaner et al., 2009; Nilsen, Kaner \& Babor, 2008). Com base nessas evidências, em vários países iniciou-se um projeto multicêntrico internacional, com o objetivo de avaliar o processo de implementação, em serviços de APS, de um modelo que consistia na detecção de usuários de risco de álcool, utilizando o Audit, seguido por uma intervenção breve de sessão única (Ronzani, Ribeiro, Amaral \& Formigoni, 2005).

No entanto, alguns estudos mostram que, apesar da eficácia da intervenção breve e da aplicabilidade do instrumento de rastreamento Audit, existem dificulda-

210 des para implantar programas de prevenção e promo- ção de saúde relacionados ao álcool. Em Juiz de Fora, pesquisa realizada com profissionais de APS, mostrou algumas dessas limitações: dificuldade para trabalhar em equipe, pouca motivação dos profissionais, alta rotatividade dos mesmos, sobrecarga de trabalho, falta de infraestrutura adequada, burocratização do trabalho e falta de organização das equipes (Ronzani et al., 2005), resultado semelhante ao obtido nas atividades desenvolvidas na Zona da Mata Mineira (Ronzani et al., 2009).

Diante desse contexto, percebe-se que muitos dos obstáculos relacionados à implantação estão ligados a fatores organizacionais, como, por exemplo, dificuldades nos trabalhos em equipe e organização dos serviços, em detrimento da aplicabilidade e eficácia das estratégias propostas. Em alguns países, foram desenvolvidos estudos que apontam uma relação entre fatores organizacionais e qualidade dos serviços de saúde, destacando-se a associação entre Clima Organizacional (CO) e os cuidados oferecidos pelos profissionais (Gershon et al., 2007; Glisson et al., 2008; MacDavitt, Chou \&Stone, 2007).

Pode-se entender clima organizacional como a percepção formal ou informal de políticas, práticas e procedimentos organizacionais (Reichers \& Schneider, 1990), sendo um fator de influência na eficiência dos resultados, bem como na conduta das pessoas que fazem parte da organização (Joyce \& Slocum, 1990). Destaca-se que, nas organizações com um clima organizacional favorável, as atividades tornam-se mais confortáveis e fáceis, produzindo maior satisfação no trabalho e obtendo maior potencial dos trabalhadores (Sánchez et al., 2006).

Alguns estudos em setores de saúde mostram um relacionamento positivo entre clima organizacional e a competência de profissionais dos setores de enfermagem (Mok \& Au-Yeung, 2002; Ying, Kunaviktikul \& Tonmukayakal, 2007).

Em serviços de saúde mental, essa relação também se destaca como fator de influência nos cuidados oferecidos aos pacientes. Esses estudos indicam que a eficiência organizacional depende de diversas características do contexto, incluindo cultura, estrutura, clima e atitudes no trabalho (Glisson et al., 2008).

Apesar da relevância do tema, poucos estudos abordam especificadamente o CO e a qualidade dos 
serviços oferecidos na APS (Ohaman-Strickland et al., 2007; Sánchez et al., 2006). Em relação às estratégias de prevenção ao uso de risco de álcool, essas avaliações tornam-se ainda mais restritas. Tendo em vista as diversas implicações do consumo excessivo de álcool para a saúde física, psicológica e social, conforme descrito inicialmente, faz-se necessário investigar de forma ampla a influência de fatores organizacionais na efetividade de tais estratégias na APS.

O presente artigo teve como objetivo avaliar a associação do clima organizacional às práticas de prevenção ao uso de risco de álcool, entre profissionais de APS da Zona da Mata Mineira (MG), buscando um maior conhecimento sobre os fatores que influenciam na qualidade dos serviços oferecidos em APS, bem como a possibilidade de se estabelecerem estratégias de prevenção mais eficazes.

\section{Método}

Participaram do estudo 97 profissionais de 3 municípios da Zona da Mata Mineira, cujos nomes optou-se por não revelar, por razões éticas. A amostragem foi composta por 54 profissionais do município A, 35 do município B e 8 do município C, dentre eles 6 médicos, 10 enfermeiros, 12 técnicos/auxiliares de enfermagem, 64 agentes comunitários de saúde e 5 outros profissionais.

O trabalho faz parte do projeto "Disseminação de Práticas de Prevenção ao Uso de Risco de Álcool em Serviços de APS da Zona da Mata Mineira" e foi aprovado pelo Comitê de Ética em Pesquisa da Universidade Federal de São Paulo (0419/06). Todos os participantes do estudo assinaram o termo de consentimento livre e esclarecido, em conformidade com as normas da instituição.

Para melhor organização das atividades, as ações foram desenvolvidas em três etapas: 1) sensibilização para os problemas relacionados ao uso de risco de álcool; 2) treinamento para práticas de prevenção, com aplicação de Audit e realização de intervenção breve; 3) avaliação de processo.

Em um primeiro momento, foram feitos contatos iniciais com gestores (Secretários de Saúde), profissionais de saúde e comunidade, para sensibilização sobre o problema do uso de álcool em cada município e obtenção de colaboradores no desenvolvimento das atividades.

Posteriormente, foram desenvolvidos dois treinamentos, com carga horária presencial de 8 horas, distribuídas nos tópicos: a) noções e conhecimentos básicos sobre o uso de álcool e suas consequências para a saúde pública; b) utilização de instrumentos de detecção (Audit); c) utilização da técnica de intervenção breve para os diversos níveis de uso de álcool; d) como implementar as práticas de triagem e intervenção breve nos serviços.

Após a capacitação presencial, deu-se seguimento ao treinamento com acompanhamento das equipes do Programa Saúde da Família (PSF) durante seis meses. No primeiro mês foram realizados encontros semanais com as equipes e, posteriormente, mensais, com a finalidade de acompanhar a rotina das Unidades e discutir a aplicação da técnica na realidade de cada serviço, baseando-se no princípio da Educação Permanente em Saúde.

A avaliação de processo foi feita utilizando metodologia quantitativa, sendo este estudo resultado de uma das avaliações realizadas.

Para o presente estudo, foram utilizados questionários e escalas, respondidos pelos profissionais em forma de auto-relato, e aplicados 6 meses após o treinamento. Os questionários foram respondidos durante o horário do trabalho, com acompanhamento dos pesquisadores. Como indicadores de realização de atividades de prevenção ao uso de álcool foram definidos o número de Audits aplicados e de intervenções breves realizadas pelos profissionais, registrados no período de 6 meses. Outro indicador utilizado para avaliar as práticas de prevenção foi definido a partir de perguntas específicas sobre frequência e motivação para a realização de atividades de prevenção ao uso de álcool. Segue abaixo a descrição dos instrumentos utilizados:

- Escala de clima organizacional: escala utilizada para avaliar a percepção dos profissionais de APS sobre atributos organizacionais relevantes para práticas de saúde da família, como, por exemplo, sua percepção sobre o papel da liderança, a carga de trabalho e a comunicação entre a equipe. Era composta por 18 itens, com respostas tipo Likert ( 1 = discordo totalmente, 2 = dis- 
cordo, 3 = neutro, 4 = concordo, 5 = concordo totalmente) e incluía as subescalas:Tomada de Decisão, Caos/ Stress e Comunicação (Ohaman-Strickland et al., 2007).

- Número de Audits aplicados e intervenções breves realizadas: o número de Audits e intervenções breves foi contabilizado durante os 6 meses de acompanhamento das atividades. Em todas as reuniões (semanais e mensais), os profissionais apresentavam os instrumentos aplicados para registro de acompanhamento. Para acompanhamento das intervenções breves foram realizados repasses semanais de todos os Audits aplicados por profissional, sendo questionado sobre a realização de intervenção após aplicação de cada instrumento. Nesse caso, considerou-se como intervenção breve qualquer orientação sobre o padrão de consumo de álcool ou sobre os danos associados ao uso de risco da substância.

- Prática de prevenção ao uso de álcool: questionário estruturado, composto de cinco questões, abordando especificamente a prática geral de atividades de prevenção e promoção de saúde; a motivação para realizar trabalhos de prevenção ao uso nocivo de álcool; a frequência com que o profissional pergunta para os pacientes sobre seu consumo de álcool; a frequência com que os profissionais aconselham pacientes com problemas relacionados ao álcool para diminuir ou parar o consumo; a prontidão para ação para realizar trabalho de prevenção ao uso nocivo de álcool.

Em relação à análise dos dados, as informações foram todas organizadas e digitadas em software estatístico para exame das variáveis. Primeiramente, os dados foram submetidos aos procedimentos de estatística descritiva para avaliar as variáveis estudadas em termos de distribuições de frequências, médias e desvio-padrão. A média de cada escala foi obtida através da somatória das respostas, ponderada pelo número total de itens em cada uma das três escalas.

A partir de então, verificou-se através do teste estatístico de Kolmogorov-Smirnov a hipótese de distribuição não normal dos escores brutos nas subescalas de clima, nas questões do questionário sobre práticas dos profissionais de PSF e na aplicação de Audits e realização de intervenções breves para a amostra estudada. A hipótese nula foi rejeitada; portanto, prosseguiu-se a análise inferencial dos dados através de estatísticas não

212 paramétricas.
Para avaliar a correlação entre clima organizacional e a prática de atividades de prevenção, utilizou-se o teste não paramétrico de Spearman, adotando-se um intervalo de confiança de $95 \%(p<0,05)$ para rejeição da hipótese nula de que maiores pontuações nas subescalas de clima relaciona-se com menor envolvimento dos profissionais em práticas de prevenção ao uso de risco de álcool.

\section{Resultados}

Os resultados relacionados com a média de idade, gênero, categoria profissional, tempo de profissão e escolaridade estão descritos na Tabela 1. Observou-se uma diferença significativa entre os gêneros (80,4\% do sexo feminino), categoria profissional (66\% agente comunitário de saúde; 12,4\% auxiliar/técnico de enfermagem; 10,3\% enfermeiro; 6,2\% médico e 5,2\% outros profissionais) e escolaridade (63,9\% apresentavam ensino médio completo; 25,8\% superior completo; 4,1\% ensino médio incompleto; 4,1\% superior incompleto e 2,1\% ensino fundamental completo). A idade média dos profissionais foi 32,86 anos (Desvio-Padrão - DP=10,5 anos), e o tempo médio de atuação em PSF foi 3,64 anos (DP=4,64 anos).

Tabela 1. Descrição da amostra estudada para as variáveis sexo, idade, categoria profissional, tempo de profissão e escolaridade (n=97). Zona da Mata Mineira (MG), 2007.

\begin{tabular}{lcc}
\hline Sexo & $\mathrm{n}$ & $\%$ \\
Feminino [n (\%)] & 78 & 80,4 \\
Idade [M (DP)] & $\mathrm{M}=32,86, \mathrm{DP}=10,5$ \\
Categoria profissional [n (\%)] & $\mathrm{n}$ & $\%$ \\
Agente comunitário de saúde & 64 & 66 \\
Auxiliar/Técnico de enfermagem & 12 & 12,4 \\
Enfermeiro & 10 & 10,3 \\
Médico & 6 & 6,2 \\
Outros profissionais & 5 & 5,2 \\
Tempo de profissão (em anos) [M (DP)] & $\mathrm{M}=3,64, \mathrm{DP}=4,64$ \\
Escolaridade [n (\%)] & $\mathrm{n}$ & $\%$ \\
Médio completo & 62 & 63,9 \\
Superior completo & 25 & 25,8 \\
Médio incompleto & 4 & 4,1 \\
Superior incompleto & 4 & 4,1 \\
Ensino fundamental completo & 2 & 2,1 \\
\hline
\end{tabular}

M: média; DP: desvio-padrão. 


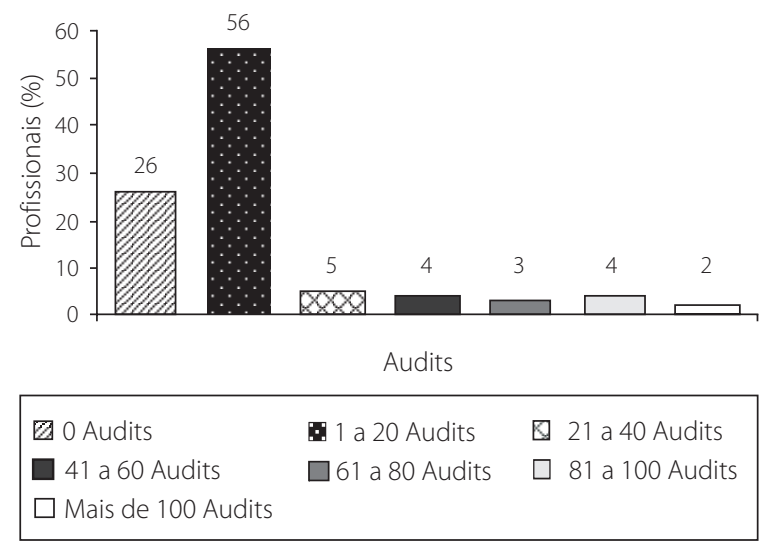

Figura 1. Distribuição de Audits aplicados pelos profissionais. Nota: Audits: alcohol use disorders identification tests.

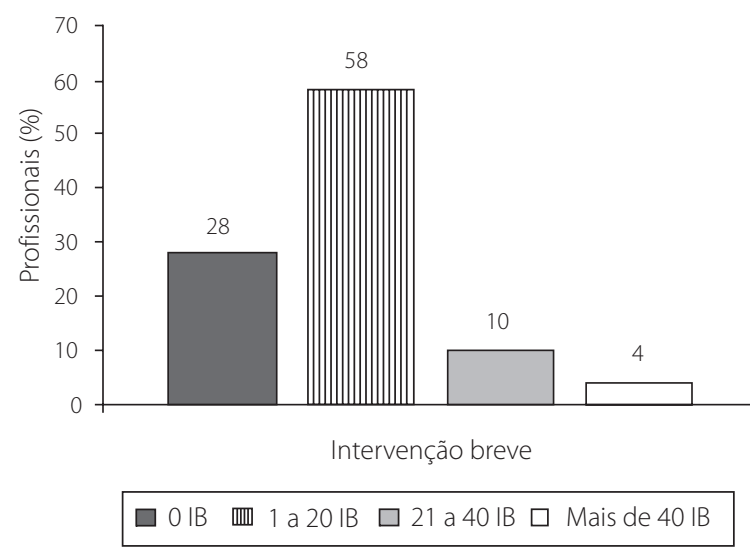

Figura 2. Distribuição de intervenções breves realizadas pelos profissionais.

Nota: IB: intervenção breve.
Foram aplicados 1472 Audits e realizadas 941 intervenções breves, no período de seis meses de acompanhamento das atividades. As Figuras 1 e 2 mostram a quantidade de instrumentos aplicados por profissional. A maior porcentagem da amostra aplicou de 1 a 20 Audits (56\%) e realizou de 1 a 20 intervenções breves (58\%). Uma pequena porcentagem (2\% para Audit e 4\% para intervenção breve) aplicou mais de 100 instrumentos e realizou mais de 40 intervenções breves, contrastando com $26 \%$ da amostra que não aplicou nenhum instrumento, e 28\% que não realizou nenhuma intervenção breve.

Os principais resultados na escala de CO foram apresentados por dimensão na Tabela 2. A maior pontuação média foi na dimensão Tomada de Decisão, com o valor de 3,47 ( $D P=0,52)$, em escala composta de 8 itens e com altos escores, descrevendo uma prática participativa, em que os resultados são estimulados pela liderança. A dimensão Comunicação foi composta de 4 itens, obtendo média de 3,43 ( $D P=0,63)$, sendo que escores elevados descrevem uma boa comunicação entre os profissionais. A subescala Caos/Stress obteve pontuação média de 3,14 (DP=0,61), observando-se que altos escores nessa subescala indicam sobrecarga de trabalho.

Na correlação entre CO e práticas de prevenção ao uso de risco de álcool, obteve-se correlação significativa entre as dimensões da escala de CO e o número de Audits aplicados e intervenções breves realizadas (Tabela 3). A correlação mais forte ocorreu entre Tomada de Decisão e número de intervenções breves realizadas ( $r=0,337 ; p=0,001)$, e, em seguida a correlação da mesma subescala com o número de Audits aplicados ( $r=0,288$;

Tabela 2. Dados descritivos para cada dimensão da escala de clima organizacional (CO), Média (M), Desvio-Padrão (DP), valor máximo e valor mínimo avaliados a partir de escala Likert de 5 pontos. Zona da Mata Mineira (MG), 2007.

\begin{tabular}{|c|c|c|c|c|c|c|}
\hline Dimensão escala CO & $N^{\circ}$ de itens & Descrição & M & DP & $\begin{array}{l}\text { Valor } \\
\text { máximo }\end{array}$ & $\begin{array}{l}\text { Valor } \\
\text { mínimo }\end{array}$ \\
\hline Tomada de decisão & 8 & $\begin{array}{l}\text { Altos escores descrevem uma Tomada de Decisão } \\
\text { participativa, na qual os resultados são estimulados pela } \\
\text { liderança. }\end{array}$ & 3,47 & 0,52 & 4,5 & 1,88 \\
\hline Comunicação & 4 & $\begin{array}{l}\text { Altos escores sugerem melhor comunicação entre os } \\
\text { profissionais. Os problemas são resolvidos através de } \\
\text { discussões em equipe ou consultas recíprocas. }\end{array}$ & 3,43 & 0,63 & 4,75 & 2,0 \\
\hline Caos/stress & 6 & $\begin{array}{l}\text { Altos escores indicam que os profissionais se sentem } \\
\text { sobrecarregados pela demanda de trabalho. }\end{array}$ & 3,14 & 0,61 & 4,83 & 1,67 \\
\hline
\end{tabular}


Tabela 3. Correlação de Spearman entre o número de Audits aplicados, IB realizadas e dimensões da escala de clima organizacional ( $\mathrm{n}=97$ ). Zona da Mata Mineira (MG), 2007.

\begin{tabular}{llcl}
\hline & & Número de Audits aplicados por profissional & Número de IB realizadas por profissional \\
\hline Tomada de Decisão & Coeficiente de Correlação & $0,288^{* *}$ & $0,337^{* *}$ \\
& Sig (2-caudas) & 0,004 & 0,001 \\
Comunicação & Coeficiente de Correlação & $0,215^{* *}$ & 0,035 \\
& Sig. (2-caudas) & $0,281^{* *}$ \\
Caos/Stress & Coeficiente de Correlação & 0,082 & 0,05 \\
& Sig (2-caudas) & 0,424 & 0,021 \\
\hline
\end{tabular}

${ }^{* *} p<0,05$; IB: intervenção breve.

p=0,004). Tomada de Decisão também teve correlação significativa com a prática em atividade geral de prevenção e promoção de saúde $(r=0,220 ; p=0,030)$. A subescala Comunicação relacionou-se positivamente com as intervenções breves realizadas $(r=0,281 ; p=0,005)$ e com o número de Audits aplicados ( $r=0,215 ; p=0,035)$. Não houve correlação entre a aplicação de Audits e intervenções breves realizadas com a dimensão Caos/Stress. As demais questões utilizadas como indicadores de práticas de prevenção ao uso de risco de álcool não se relacionaram significativamente com as subescalas de $\mathrm{CO}$.

\section{Discussão}

Este estudo avaliou fatores organizacionais importantes que podem influenciar a prática dos profissionais da APS para o desenvolvimento de atividades de prevenção ao uso de risco de álcool. Os resultados mostraram que existe uma correlação significativa entre as escalas de $\mathrm{CO}$ e atividades de prevenção (aplicação de Audit e realização de intervenção breve). Tomada de Decisão e Comunicação relacionaram-se mais fortemente com o desenvolvimento das atividades.

Os dados obtidos sugerem que os profissionais aplicam mais Audits e realizam mais intervenções breves quando percebem que a liderança estimula resultados, quando as decisões são tomadas de forma participativa e quando a comunicação entre a equipe favorece a solução de problemas. Esses dados destacam a o papel importante dos coordenadores de equipe na estruturação de um ambiente de trabalho produtivo e de qualidade, oferecendo suporte técnico e estimulando a participação efetiva dos profissionais nas decisões 214 tomadas, além de criar condições para o aprimoramento de competências. O papel da comunicação também chama atenção para a importância das atividades em PSF serem realizadas de forma conjunta, com o apoio mútuo para superação de dificuldades e obstáculos presentes na prática. Essa questão é relevante para se pensarem formas de implementar estratégias de prevenção ao uso de risco de álcool, que consigam envolver a equipe como um todo, já que muitas vezes o desenvolvimento das atividades se limita à atuação dos agentes comunitários (Ronzani et al., 2009), reforçando uma prática desintegrada e oposta aos objetivos preconizados nos trabalhos em equipe de saúde da família.

Os resultados encontrados neste estudo são novos para a discussão sobre a efetividade de estratégias de prevenção ao uso de risco de álcool. Muitos estudos enfocam o desenvolvimento de instrumentos de rastreamento para o consumo de drogas e a avaliação de intervenções breves nessa problemática (Humeniuk et al., 2008; Kaner et al., 2009). No entanto, poucas pesquisas abordam os diversos fatores organizacionais que envolvem a prática dos serviços e que podem atuar como facilitadores (ou dificultadores) na transferência, para a rotina profissional, de conhecimentos adquiridos em capacitações presenciais (Babor, Higgins-Biddle, Dauser, Higgins \& Burleson, 2005; Pidd et al., 2004).

A avaliação do clima organizacional é uma das formas comumente utilizadas para mensurar a percepção dos trabalhadores em relação a fatores do ambiente de trabalho. No Brasil, embora se encontrem instrumentos elaborados e adaptados para avaliar o clima em diferentes setores da economia (Crespo, 2004; Martins, Oliveira, Silva, Pereira \& Sousa, 2004), o mesmo não se dá na área de saúde, em que as opções são mais restritas, ressalvada a literatura internacional (Sánchez 
et al., 2006; Ohaman-Strickland et al., 2007; Wienand, Cinotti, Nicoli \& Bisagni, 2007).

Em 2009, posteriormente à coleta de dados deste estudo, houve a validação da primeira escala para avaliar o clima organizacional em setores de saúde brasileiros, priorizando aspectos peculiares desse tipo de serviço, tal como o relacionamento com a comunidade (Menezes, Sampaio, Gomes, Teixeira \& Santos, 2009).

Em contrapartida, em vários países, além de se observar maior investimento em instrumentos específicos para avaliar o CO em setores de saúde, encontram-se também diversos estudos associativos avaliando a correlação entre o clima e atividades gerais em saúde (MacDavitt et al., 2007). Estudo realizado em hospital universitário na China encontrou uma correlação positiva entre $\mathrm{CO}$ e a competência de enfermeiras $(p<0,01 ; r=0,41)$, sendo estas avaliadas em sete dimensões: desenvolvimento profissional, cuidados clínicos, ensinamento/treinamento, prática ética e legal, pensamento crítico e atitude em pesquisa, liderança e relacionamento interpessoal (Ying et al., 2007). Resultado semelhante foi obtido em pesquisa desenvolvida em atendimentos de emergência nos Estados Unidos, indicando que os profissionais que vêem suas condições de trabalho positivamente fornecem melhores intervenções aos pacientes com problemas psiquiátricos (Wright, Linde, Rau, Gayman \& Viggiano, 2003).

Cabe destacar que uma diferenciação importante foi obtida na correlação entre CO e atividades de prevenção. As escalas Tomada de Decisão e Comunicação correlacionaram-se mais fortemente com a realização de intervenção breve do que com Audit (Tabela 3). Talvez essa diferença se relacione com o fato de a intervenção breve ser uma técnica mais próxima das atividades que os profissionais de PSF desenvolvem cotidianamente. Muitos deles provavelmente já haviam realizado algo semelhante, mesmo antes da capacitação, sem no entanto denominar essas atividades como intervenção breve. Além disso, a intervenção breve é mais fácil de adequar à realidade de trabalho das equipes, o que facilita a maior inserção dessa técnica como rotina dos profissionais. Já o Audit, por ser um instrumento padronizado, diferencia-se muito do material habitualmente utilizado. Muitos profissionais provavelmente já tinham perguntado para os pacientes sobre o consumo de bebidas alcoólicas, porém não se baseavam em um instrumento padronizado e estruturado.
Essa dificuldade dos profissionais em lidar com materiais novos, como por exemplo, o Audit, reforça a importância da liderança e da cooperação do grupo na superação das dificuldades apresentadas como barreiras à implementação das estratégias propostas. Nesse sentido, diversas pesquisas indicam que o trabalho em equipe (Ying et al., 2007; Wright et al., 2003) e o suporte da liderança (Mok \& Au-Yeung, 2002) são os fatores do clima organizacional que se correlacionam fortemente com a qualidade e resultado das atividades realizadas pelos profissionais. Igualmente, estudo desenvolvido com 213 equipes de APS na Catalunha (Espanha) mostra que fatores organizacionais, como cooperação e facilitação em grupo, estão entre os pontos que melhor explicam os resultados do trabalho em medicina da família (Gené-Badia et al., 2007).

É importante ressaltar que, apesar de os profissionais terem percebido o ambiente de trabalho com uma sobrecarga de atividades (Tabela 2), não houve correlação entre a subescala Caos/Stress e o desempenho naquelas atividades (Tabela 3). Esse resultado sugere que a alta demanda de serviços, frequentemente citada como obstáculo para o desenvolvimento de atividades na APS (Ronzani et al., 2009), não interferiu na prática da equipe quanto às atividades propostas, ao contrário dos resultados obtidos nas escalas Comunicação e Tomada de Decisão.

Os resultados alcançados neste estudo sugerem que os fatores que envolvem o ambiente de trabalho são aspectos importantes no desenvolvimento de atividades na atenção primária à saúde, donde a necessidade de se ampliarem as discussões sobre a prática e a qualidade desses serviços.

\section{Considerações Finais}

Este estudo objetivou avaliar a influência do clima organizacional na prática de prevenção ao uso de risco de álcool entre profissionais da Atenção Primária à Saúde, a partir de três aspectos: Tomada de Decisão, Comunicação e Caos/Stress. Os resultados sugeriram que equipes que percebem um bom ambiente de trabalho tendem a ter maior comprometimento nas atividades. A liderança, o envolvimento dos profissionais na tomada de decisão e a comunicação dentro do grupo foram aspectos que se destacaram. Os resultados 
são ainda iniciais, porém trazem reflexões importantes para se pensar o papel do trabalho em equipe e dos coordenadores de PSF como facilitadores do desenvolvimento das atividades. Essa questão se coloca como um desafio diante da grande rotatividade de profissionais e gestores, especialmente porque o problema demanda trabalho em equipe estável. Dessa forma, este estudo foi uma tentativa de ampliar o conhecimento sobre os fatores que podem influenciar a prática dos profissionais de PSF em atividades de prevenção ao uso de risco de álcool, buscando trazer contribuições para se traçarem estratégias mais efetivas.

Cabe destacar que o próprio contexto de trabalho das equipes de PSF se coloca como uma limitação desta pesquisa. A rotatividade dos profissionais restringe o número de participantes neste tipo de desenho metodológico, tendo em vista que o número inicial de sujeitos não se estabelece até a fase final do processo, trazendo implicações não só para a pesquisa como também para a implementação das atividades como rotina das equipes. Além do mais, em função das visitas domiciliares, que são ações estruturantes do Programa Saúde da família, muitas vezes os pesquisadores tinham dificuldade em acompanhar as equipes completas nas reuniões semanais ou mensais, tendo em vista a preocupação de não alterar a rotina de trabalho dos profissionais.

Dessa forma, destaca-se a necessidade de se desenvolverem estudos complementares, com um maior número de equipes de PSF, possibilitando a verificação mais adequada de hipóteses e a generalização dos resultados. Além disso, sugere-se a utilização de ferramentas validadas para avaliar o clima organizacional em setores de saúde brasileiros, contribuindo para avaliações mais detalhadas e apontando de forma mais precisa os fatores que melhor se correlacionam com a prática dos profissionais de APS.

\section{Referências}

Babor, T. F., \& Higgins-Biddle, J. C. (2001). Brief intervention for hazardous and harmful drinking: a manual for use in Primary Care. Geneva: World Health Organization.

Babor, T. F., Higgins-Biddle, J., Dauser, D., Higgins, P., \& Burleson, J. (2005). Alcohol screening and brief intervention in primary care settings: implementation models and predictors. Journal of Studies on Alcohol, 66 (3), 361-368.
Babor, T. F., Higgins-Biddle, J. C., Saunders, J. B., \& Monteiro, M.G. (2001). AUDIT- the alcohol use disorders identification test: guidelines for use in primary care. Geneva:World Health Organization.

Bertholet, N., Daeppen, J. B., Wietlisbach, V., Fleming, M., \& Burnand, B. (2005). Reduction of alcohol consumption by brief alcohol intervention in primary care: systematic review and meta-analysis. Archives of Internal Medicine, 165 (9), 986-995.

Crespo, M. L. F. (2004). Construção de uma medida de clima criativo em organizações. Estudos de Psicologia (Campinas), 21 (2), 91-99. doi: 10.1590/S0103-166X2004000200007.

Gené-Badia, J., Ascaso, C., Escaramis-Babiano, G., SampietrColom, L., Catalán-Ramos, A., Sans-Corrales, M., et al. (2007). Personalised care, acess, quality and team coordination are the main dimensions of family medicine output. Family Practice, 24 (1), 41-47.

Gershon, R. R. M., Stone, P. W., Zeltser, M., Faucett, J., MacDavitt, K., \& Chou, S-S. (2007). Organizational climate and nurse health outcomes in the United States: a sistematic review. Industrial Health, 45 (5), 622-636.

Glisson C., Landsverk J., Schoenwald S., Kelleher K., Hoagwood K. E, Mayberg S., et al. (2008). Assessing the organizational social context (OSC) of mental health services: implications for research and practice. Administration and Policy Mental Health and Mental Health Services Research, 35, 98-113.

Humeniuk, R., Ali, R., Babor, T. F., Farrell, M., Formigoni, M. L., Jittiwutikarn, J., et al. (2008). Validation of the alcohol, smoking and substance involvement screening test (ASSIST). Addiction Research Report, 103 (6), 1039-1047.

Joyce, W. E., \& Slocum, J. W. (1990). Strategic context and organizational climate. In B. Schneider (Org), Organizational climate and culture (pp.130-150). San Francisco: Jossey-Bass.

Kaner, E. F. S., Dickinson, H. O., Beyer, F., Pienaar, E., Schlesinger, C., Campbell, F., et al. (2009). The effectiveness of brief alcohol interventions in primary care settings: a systematic review. Drug and Alcohol Review, 28 (3), 301-323.

MacDavitt, K., Chou, S-S., \& Stone, P. W. (2007). Organizational climate and health care outcomes.joint commission journal on quality and patient safety, 33 (11), 45-56.

Martins, M. C. F., Oliveira, B., Silva, C. F., Pereira, K. C., \& Sousa, M. R. (2004). Construção e validação de uma escala de medida de clima organizacional. Psicologia: Organizações eTrabalho, 4 (1), 37-60.

Menezes, I. G., Sampaio, L. R., Gomes, A. C. P., Teixeira, F. S., \& Santos, P. S. (2009). Escala de clima organizacional para organizações de saúde: desenvolvimento e estrutura fatorial. Estudos de Psicologia (Campinas), 26 (3), 305-316. doi: 10.1590/S0103-166X2009000300004.

Mok, E., \& Au-Yeung, B. (2002). Relationship between organizational climate and empowerment of nurses in Hong Kong. Journal of Nursing Management, 10 (3): 129-137.

Nilsen, P., Kaner, E., \& Babor, T. (2008). Brief intervention, three decades on: an overview of research findings and strategies for more widespread implementation Nordic Studies on Alcohol and Drugs, 25 (6), 453-567. 
Ohaman-Strickland, P. A., Orzano, A. J., Nutting, P. A., Dickinson, W. P., Scott-Cawiezell, J., Hahn, K., et al. (2007). Measuring organizational attributes of primary care practices: development of a new instrument. Health Research and Educational Trust, 42 (3), 1257-273.

Pidd, K., Freeman, T., Skinner, N., Addy, D., Shoobridge, J., \& Roche, A. M. (2004). From training to work practice change: an examination offactors Influencing training transfer in the alcohol and otherdrugs Field. Australia: National Centre for Education and Training on Addiction.

Reichers, A. E., \& Schneider B. (1990). Climate and culture: an evolution of constructs. In B. Schneider (Org.), Organizational climate and culture (pp.5-39). San Francisco: Jossey-Bass.

Ronzani, T., Mota, D. C. B., \& Souza, I. C. W. (2009). Prevenção do uso de álcool na atenção primária em municípios do estado de Minas Gerais. Revista de Saúde Pública, 43 (1), 51-61.

Ronzani, T., Ribeiro, M. S., Amaral, M. B., \& Formigoni, M. L. O. (2005). Implantação de rotinas de rastreamento do uso de risco de álcool e de uma intervenção breve na atenção primária à saúde: dificuldades a serem superadas. Cadernos de Saúde Pública, 21 (3), 852-861.
Sánchez, A. D., Saameño, J. A. B., López-Montes, M. T. M. C., Castillo, L., Fernández, L. A. L., \& Claret, P. L. (2006). Las dimensiones del clima organizacional percibidas por los médicos de familia. Atención Primaria, 37 (9), 489-97.

Ying, L., Kunaviktikul, W., \& Tonmukayakal, O. (2007). Nursing competency and organizational climate as perceived by staff nurses in a Chinese university hospital. Nursing and Health Sciences, 9 (3), 221-227.

Wienand, U., Cinotti, R., Nicoli, A., \& Bisagni, M. (2007). Evaluating the organisational climate in Italian public healthcare institutions by means of a questionnaire. BMC Health Services Research, 73 (7),1-13.

World Health Organization. (2007). Expert Committee on problems related to alcohol consumption. Geneva: World Health Organization.

Wright, E. R., Linde, B., Rau, N. L., Gayman, M., \& Viggiano, T. (2003). The effect of organizational climate on the clinical care of patients with mental health problems. Journal of Emergency Nursing, 29 (4), 314-321.

Recebido em: 30/4/2010

Versão final reapresentada em: 25/10/2010

Aprovado em: 16/12/2010 
